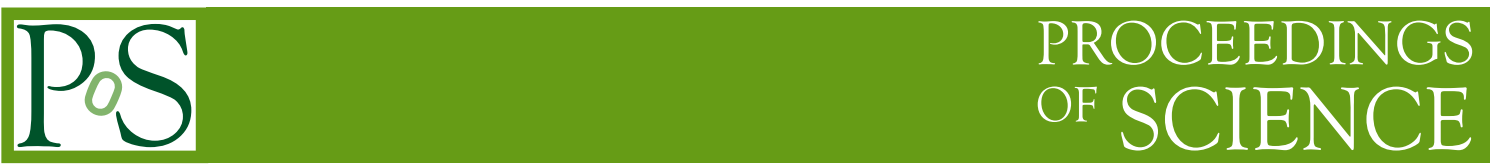

\title{
Measuring High-Energy Spectra with HAWC
}

\author{
S. S. Marinelli ${ }^{a}$ and J. A. Goodman ${ }^{* b}$ for the HAWC Collaboration ${ }^{\dagger}$ \\ ${ }^{a}$ Michigan State University, United States \\ Email: marine20@msu.edu \\ ${ }^{b}$ University of Maryland, United States \\ Email: goodman@umdgrb.umd.edu
}

The High-Altitude Water-Cherenkov (HAWC) experiment is a $\mathrm{TeV} \gamma$-ray observatory located $4100 \mathrm{~m}$ above sea level on the Sierra Negra mountain in Puebla, Mexico. The detector consists of 300 water-filled tanks, each instrumented with 4 photomultiplier tubes that utilize the waterCherenkov technique to detect atmospheric air showers produced by cosmic $\gamma$ rays. Construction of HAWC was completed in March of 2015. The experiment's wide instantaneous field of view ( 2 sr) and high duty cycle (> 95\%) make it a powerful survey instrument sensitive to pulsars, supernova remnants, and other $\gamma$-ray sources. The mechanisms of particle acceleration at these sources can be studied by analyzing their high-energy spectra. To this end, we have developed an event-by-event energy-reconstruction algorithm using an artificial neural network to estimate energies of primary $\gamma$ rays at HAWC. We will present the details of this technique and its performance as well as the current progress toward using it to measure energy spectra of $\gamma$-ray sources.

35th International Cosmic Ray Conference-ICRC2017

10-20 July, 2017

Bexco, Busan, Korea

*Speaker.

${ }^{\dagger}$ A complete list of authors is available at http://www.hawc-observatory.org/collaboration/icrc2017.php. 


\section{Introduction}

Among the fundamental questions in particle astrophysics are those of the sources and acceleration mechanisms of the high-energy particles that are observed to arrive at the Earth from space. Theories postulating different acceleration mechanisms have implications for the distributions of energies of particles emitted at these sources and therefore can be constrained by measurements of these sources' emitted energy spectra. Making such measurements requires the precise reconstruction of particle energies by observatories sensitive to these particles.

The High-Altitude Water-Cherenkov (HAWC) detector observes $\mathrm{TeV} \gamma$ rays from an altitude of $4100 \mathrm{~m}$ in the state of Puebla, Mexico. The experiment's 300 water-filled tanks are each instrumented with 4 photomultiplier tubes (PMTs) that detect the Cherenkov light produced by $\gamma$-rayinduced extensive air showers as they pass through the tanks. Particle arrival times and deposited charges are reconstructed from PMT data, allowing estimation of various shower parameters such as zenith and azimuth angles and primary energy. A detailed description of the detector's electronics and data-acquisition system can be found at [1]. By reconstructing primary energies with sufficient precision, HAWC can constrain the spectral energy distributions (SEDs) of $\gamma$-ray sources, thereby probing their mechanisms of particle acceleration. This paper introduces a new algorithm for reconstructing the energies of HAWC $\gamma$-ray events and establishes the performance of this technique via Monte Carlo (MC) simulations and on a calibration source, the Crab Nebula.

\section{Energy-reconstruction technique}

The algorithm described herein uses an artificial neural network (NN) to reconstruct energies of photons detected by HAWC. It was implemented using the Toolkit for Multivariate Analysis [2] $\mathrm{NN}$ implementation.

An $\mathrm{NN}$ is a complicated function mapping several quantities associated with an event (input variables) to some regression target or output variable, in this case $\log _{10} E$ where $E$ is the primary energy of the shower. A detailed description of the functional form of the $\mathrm{NN}$ can be found in [2]. This function is characterized by many (479 in this implementation) free parameters called weights. The optimal values of the weights are determined in a process called training, in which the weights are optimized using an MC simulation of the detector. The training process is described in Section 2.2.

The energy algorithm consists of two NNs: one for low-multiplicity events and one for highmultiplicity events. This binning has been found to yield better performance than a single NN even though multiplicity variables are included among the inputs.

\subsection{Input variables}

Fifteen input variables are used to characterize the shower energy. These variables have been chosen in order to capture three qualities of the shower: the particle multiplicity in the detector, the fraction of the shower which landed outside of the detector, and the atmospheric attenuation of the shower.

The multiplicity in the detector is a crude measure of how much energy has been deposited in it and is quantified using the fraction of PMTs hit in the event, the fraction of tanks hit, and the 
normalization factor (in logarithm) of the fit of the lateral distribution of the shower. This fit is described in more detail at [3].

The distance between the reconstructed shower-core location and the center of the HAWC array is included as an input variable in order to provide information about how much of the shower is not contained within the array.

Finally, the distance through which the shower has propagated in the atmosphere is quantified in two ways: using the reconstructed zenith angle and the lateral distribution of particles in the shower, which contains information about the shower's age. The latter is provided to the NN in the form of ten input variables, the $i$ th of which is the fraction of charge deposition in the event occurring in PMTs whose distances from the shower axis, measured in the shower plane, are between $(10 \mathrm{~m}) i$ and $(10 \mathrm{~m})(i+1)$ for $i$ between 0 and 8 inclusively; the last variable is the fraction of charge deposition in PMTs more than $90 \mathrm{~m}$ from the axis.

\subsection{Training}

The goal of the training process is to choose the vector of $\mathrm{NN}$ weights $\mathbf{w}$ that minimizes the error function, defined as

$$
D(\mathbf{w}) \equiv \frac{1}{2} \sum_{i=1}^{N_{\text {events }}} u_{i}\left[\log _{10} \hat{E}\left(\mathbf{x}_{i} ; \mathbf{w}\right)-\log _{10} E_{i}\right]^{2}
$$

where $u_{i}$ is the weight of the $i$ th MC event (the event's relative importance in the training, unrelated to the NN parameters $\mathbf{w}$, which are unfortunately also called weights), $E_{i}$ is the true energy of the $i$ th event, $\mathbf{x}_{i}$ is the $i$ th event's vector of input variables, and $\hat{E}$ is the energy estimate for the event. This minimization is performed by repeatedly iterating over the sample of training events, with a small update to $\mathbf{w}$ performed for each event. The details of the Broyden-Fletcher-Goldfarb-Shanno algorithm for updating the weights can be found in [2]. The MC events are weighted (the $u_{i}$ are set) to resemble an $E^{-2}$ power-law spectrum during the training. This weighting was chosen because it produces a relatively flat RMS error between 1 and $100 \mathrm{TeV}$ (see Section 3.2).

\section{Performance on Monte Carlo}

Several other energy-reconstruction algorithms have been developed for the HAWC observatory. These are described briefly in Section 3.1, and the performance of all energy variables including the NN is described in Section 3.2.

\subsection{Other energy variables}

The energy proxy that is presently used in the standard HAWC $\gamma$-ray analysis is the fraction of PMTs hit during the event, referred to as "fraction hit" or $f_{\text {hit }}$ [3].

A likelihood-based algorithm for energy reconstruction has also been developed. This algorithm uses a likelihood function built using MC that takes into account the locations, arrival times, and deposited charges of the PMT hits that occur during the event. The hits are assumed to be statistically independent so that the likelihood is built by combining many single-hit likelihood functions. This algorithm has also been adapted for cosmic-ray energy reconstruction and is described in [4]. 
A third algorithm, called the "ground parameter" (GP), is an estimation of the photon primary energy as a function of reconstructed zenith angle and the value of the lateral distribution function at some optimal distance from the estimated shower axis. This is described in greater detail in [5].

\subsection{Performance comparison}

The performance of these energy-reconstruction techniques is evaluated using MC simulations of $\gamma$-ray showers interacting with the HAWC detector. These simulations are described in [3]. The MC events are weighted to simulate a point source transiting at $20^{\circ}$ declination with an $E^{-2.63}$ power-law energy spectrum. Analysis cuts are applied, including an $f_{\text {hit }}$ cut at 0.044 and a requirement that the reconstructed core be within the HAWC array. Background-rejection cuts are also applied in order to simulate a signal-event population resembling what would be used in an analysis of actual data.

Figure 1a shows the distribution of fraction hit as a function of true energy. Because higherenergy photons on average produce higher-multiplicity showers, fraction hit is positively correlated with primary energy. However, several factors prevent it from determining the energy precisely. Because showers are attenuated as they travel through the atmosphere, a shower coming from a higher angle from zenith or having its first interaction higher in the atmosphere will have a lower multiplity than a shower of the same primary energy coming from a lower angle from zenith or interacting lower in the atmosphere. In addition, a shower whose core is not well centered on the detector will have a lower multiplicity in the data than one that is, again assuming equal primary energies. Furthermore, because fraction hit cannot exceed 1, at sufficiently high energies, the variable saturates and loses sensitivity to the energy.

Figure $1 \mathrm{~b}$ shows the relationship between the NN variable and true energy. Because the NN includes input variables that contain information about the containment of the shower within the array and its atmospheric attenuation, it is able to achieve a better resolution than that of fraction hit. Since the NN takes into account information beyond just the particle multiplicity of the shower, it does not saturate within the range of energies thrown in the MC, which extends well beyond $100 \mathrm{TeV}$.

Figure 2 shows the RMS error in log space of the three explicit energy estimates: the NN estimate, the likelihood estimate, and the GP estimate. The RMS error is defined as

$$
\rho \equiv \sqrt{\left\langle\left(\log _{10} \hat{E}-\log _{10} E\right)^{2}\right\rangle} .
$$

The NN displays the best RMS error in all bins. The RMS error in the 46 to $68 \mathrm{TeV}$ bin is around 0.12 , indicating 0.12 decade in energy, or about $32 \%$ in linear energy space. All of the reconstruction algorithms perform poorly below around $1 \mathrm{TeV}$. This is related to the $f_{\text {hit }}$ cut: a substantial fraction of events below $1 \mathrm{TeV}$ fail this cut, and those that pass it represent upwards multiplicity fluctuations. Thus their energies tend to be overestimated.

\section{Calibration source: the Crab Nebula}

In order to verify the ability of the new energy variables to accurately measure $\gamma$-ray energy spectra, a spectral fit of the Crab Nebula has been performed using the NN and GP variables. The 


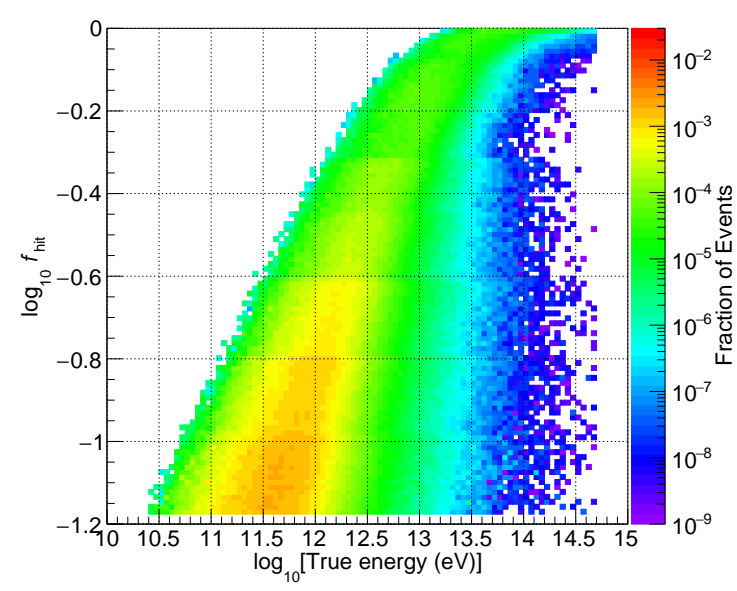

(a) Fraction hit

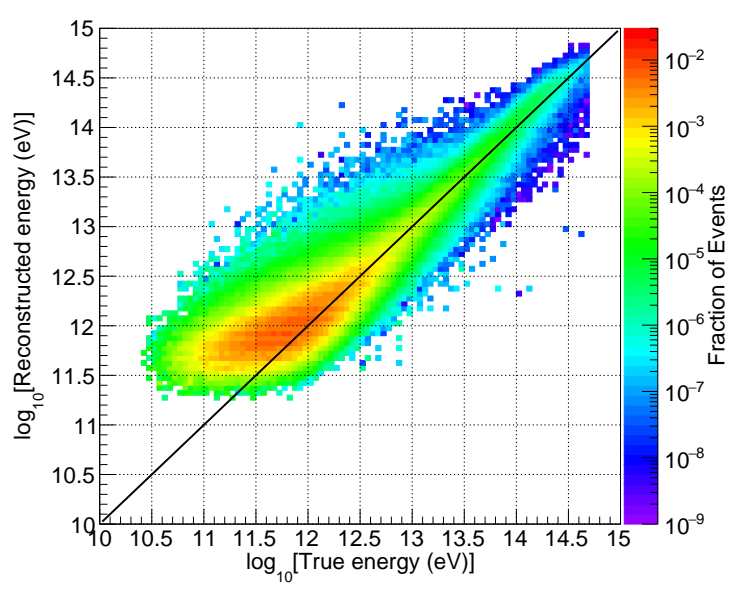

(b) NN estimator

Figure 1: Joint distribution of each of two energy variables with true energy in MC, among events passing the analysis cuts.

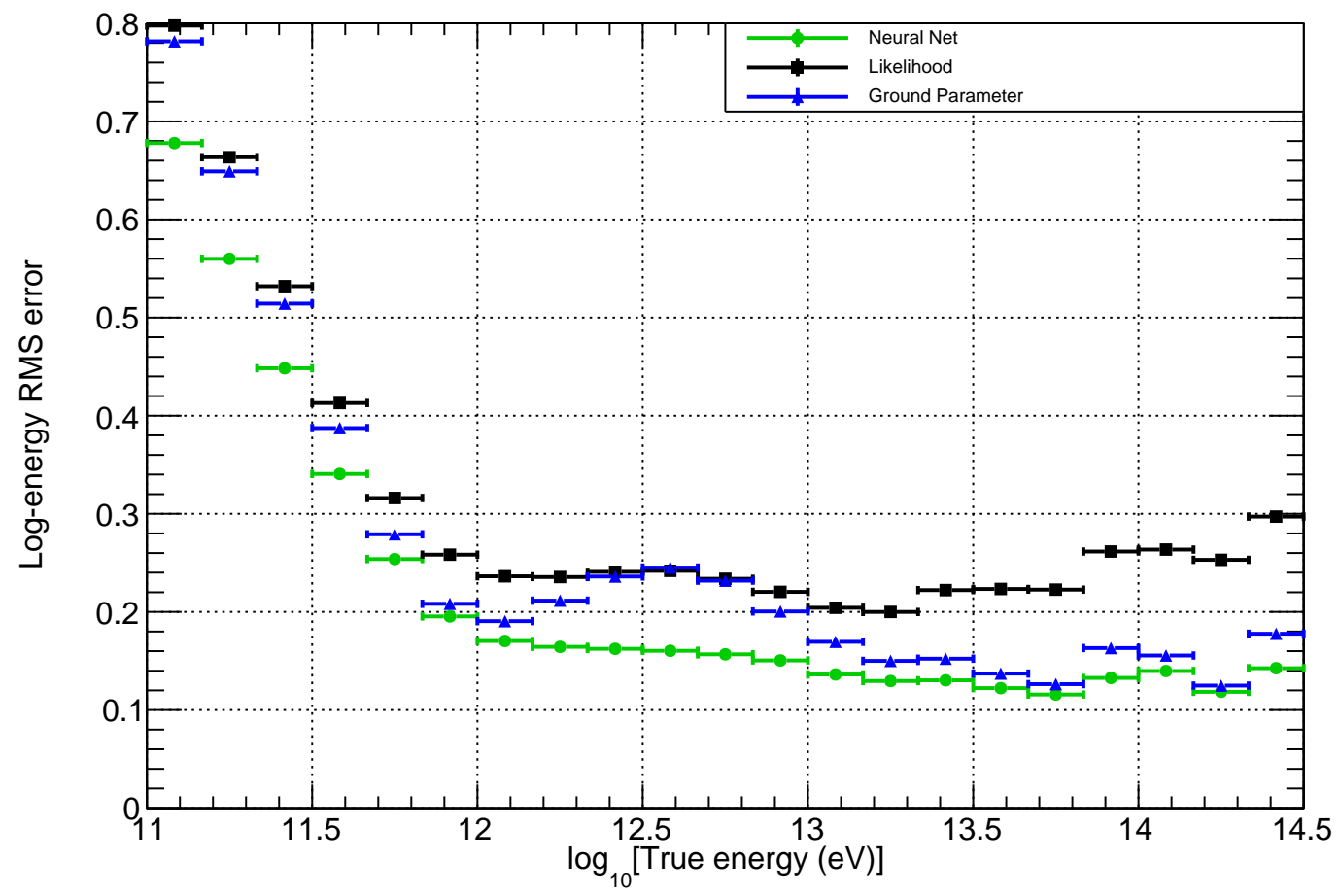

Figure 2: RMS error in log space of the NN, likelihood, and GP energy variables as a function of true energy in MC. 


\begin{tabular}{l|c|l|l|l|l|l|l} 
& & & \multicolumn{2}{|c|}{ Published HAWC } & \multicolumn{2}{|c}{ NN } & \multicolumn{2}{c}{ GP } \\
Parameter & Unit & Value & Error & Value & Error & Value & Error \\
\hline$\Phi_{0}$ & $10^{-13}\left(\mathrm{~cm}^{2} \mathrm{~s} \mathrm{TeV}\right)^{-1}$ & 2.47 & ${ }_{-0.12}^{+0.13}$ & 2.92 & 0.06 & 3.22 & 0.07 \\
$\alpha$ & & 2.627 & ${ }_{-0.035}^{+0.035}$ & 2.712 & ${ }_{-0.022}^{+0.021}$ & 2.751 & ${ }_{-0.023}^{+0.022}$ \\
$\beta$ & & 0.149 & ${ }_{-0.030}^{+0.033}$ & 0.162 & 0.015 & 0.179 & ${ }_{-0.016}^{+0.017}$
\end{tabular}

Table 1: Best-fit values and statistical uncertainties for log-parabola spectral parameters.

Likelihood energy variable is not currently used in $\gamma$-ray analyses. This was carried out via the Multi-Mission Maximum Likelihood framework, described in [6], which interacts with HAWC's Analysis and Event-Reconstruction Integrated Environment to perform a forward-folded fit of the true-energy distribution to the observed energy-estimate distribution, taking into account the joint distribution in Figure 1b. The fit was performed using 18 months of HAWC data. The Crab was modeled as a point source, with the expected distribution of reconstructed photon directions being the detector's point-spread function centered on the source's location. Its energy spectrum was assumed to be log-parabolic,

$$
\frac{d N}{d E}=\Phi_{0}\left(\frac{E}{E_{0}}\right)^{-\alpha-\beta \ln \left(E / E_{0}\right)},
$$

where $d N / d E$ is the photon particle flux, $\Phi_{0}, \alpha$, and $\beta$ are free parameters, and the pivot energy $E_{0}$ was chosen to be $7 \mathrm{TeV}$ to decorrelate the estimates of $\Phi_{0}$ and $\alpha$. Events were sorted into two-dimensional bins of estimated energy and fraction hit in the analysis; fraction hit was included because it parameterizes the detector's angular resolution better than any of the explicit energy estimates. A skymap was constructed for each bin, and a pixel-by-pixel fit was performed to find the log-parabola spectrum most compatible with the data. The result is plotted in Figure 3 along with H.E.S.S.'s reconstructed Crab spectrum from [7], and the fit spectral parameters' values are given in Table 1 along with those from the published HAWC Crab spectrum in [3].

Table 1 shows that the NN spectral fit is better able to constrain all three spectral parameters than are the fit using only $f_{\text {hit }}$ and the GP fit.

The SEDs computed using the two energy variables differ systematically from each other and from the H.E.S.S. result at some energies. An analysis of systematic errors has not yet been carried out and will be necessary in the future in order to understand this discrepancy. In particular the forward-folding technique is vulnerable to any differences in the distributions of the energy variables between data and MC, and such differences could result in the observed systematic discrepancy in the computed SEDs. The fractional systematic uncertainty on the published HAWC Crab spectral measurement is 50\% [3], which, taken as a crude estimate of the systematic error on this analysis, is sufficient to account for the difference between the SEDs measured using the two new energy variables.

\section{Conclusions}

The development of the NN energy-reconstruction method allows HAWC to resolve energies up to $100 \mathrm{TeV}$. The technique has already been put into use in measuring energy spectra of $\mathrm{TeV}$ 


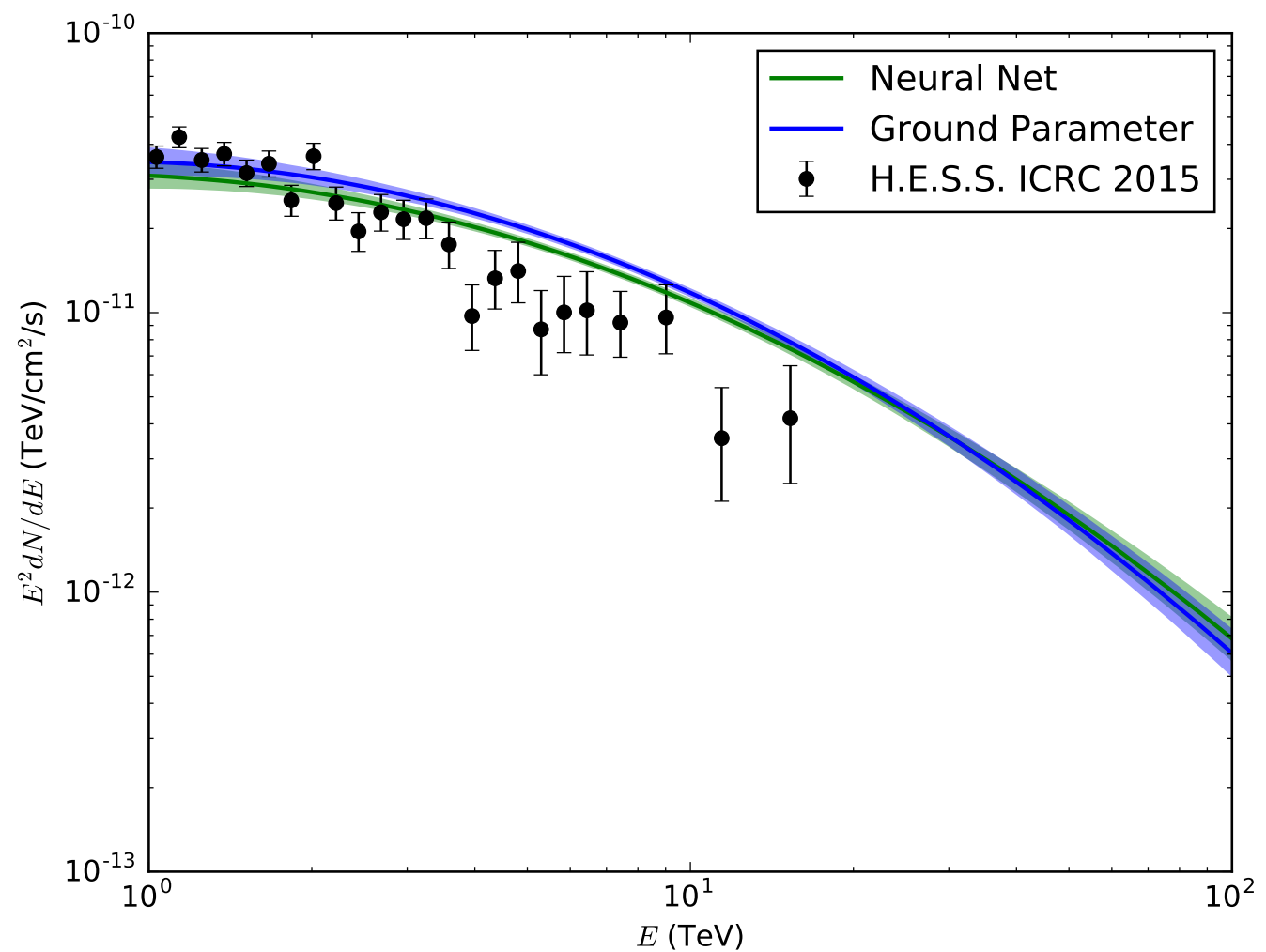

Figure 3: Preliminary fits of the Crab Nebula photon flux using the NN and GP energy variables. Shown errors on these fits are statistical only.

blazars; see [8]. Using the NN method, HAWC will be able to measure energy spectra of gammaray sources up to unprecedentedly high energies, providing a new window into the high-energy universe.

\section{Acknowledgments}

We acknowledge support from the US National Science Foundation (NSF); the US Department of Energy Office of High-Energy Physics; the Laboratory Directed Research and Development (LDRD) program of Los Alamos National Laboratory; Consejo Nacional de Ciencia y Tecnología (CONACyT), México (grants 271051, 232656, 260378, 179588, 239762, 254964, 271737, 258865, 243290, 132197), Laboratorio Nacional HAWC de rayos gamma; L'OREAL Fellowship for Women in Science 2014; Red HAWC, México; DGAPA-UNAM (grants I100317, IN111315, IN111716-3, IA102715, 109916, IA102917); VIEP-BUAP; PIFI 2012, 2013, PROFOCIE 2014, 2015; the University of Wisconsin Alumni Research Foundation; the Institute of Geophysics, Planetary Physics, and Signatures at Los Alamos National Laboratory; Polish Science Centre grant DEC-2014/13/B/ST9/945; Coordinación de la Investigación Científica de la Universidad Michoacana. Thanks to Luciano Díaz and Eduardo Murrieta for technical support. 


\section{References}

[1] A. U. Abeysekara et al., Search for TeV Gamma-Ray Emission from Point-like Sources in the Inner Galactic Plane with a Partial Configuration of the HAWC Observatory, The Astrophysical Journal $\mathbf{8 1 7}$ (2016) 3.

[2] A. Hoecker et al., TMVA: Toolkit for Multivariate Data Analysis, PoS ACAT (2007) 040, [physics/0703039].

[3] A. U. Abeysekara et al., Observation of the Crab Nebula with the HAWC Gamma-Ray Observatory, 1701.01778.

[4] HAWC collaboration, J. C. Arteaga-Velázquez et al., Estimate of the energy spectrum of the light component of cosmic rays in HAWC using the shower age and the fraction of hit PMT's, PoS ICRC2017 (2017) .

[5] K. Malone, The gamma-ray sky above $50 \mathrm{TeV}$ with the HAWC Observatory, January, 2017, http://meetings.aps.org/Meeting/APR17/Session/X4.8.

[6] G. Vianello et al., The Multi-Mission Maximum Likelihood framework (3ML), 2015, 1507.08343, https://inspirehep.net/record/1385718/files/arXiv:1507.08343.pdf.

[7] H.E.S.S. collaboration, M. Holler et al., Observations of the Crab Nebula with H.E.S.S. Phase II, PoS ICRC2015 (2016) 847, [1509.02902].

[8] HAWC collaboration, S. C. de León et al., Spectral analysis of Markarian 421 and Markarian 501 with HAWC, PoS ICRC2017 (2017). 